\title{
On the Incompressible Navier-Stokes Equations with Damping*
}

\author{
Wenyan Zhao, Zhibo Zheng" \\ Department of Mathematics, Baoshan University, Baoshan, China \\ Email: "zhengzhibo1234560911@126.com
}

Received November 23, 2012; revised February 27, 2013; accepted March 3, 2013

Copyright (c) 2013 Wenyan Zhao, Zhibo Zheng. This is an open access article distributed under the Creative Commons Attribution License, which permits unrestricted use, distribution, and reproduction in any medium, provided the original work is properly cited.

\begin{abstract}
We consider dynamics system with damping, which are obtained by some transformations from the system of incompressible Navier-Stokes equations. These have similar properties to original Navier-Stokes equations the scaling invariance. Due to the presence of the damping term, conclusions are different with proving the origin of the incompressible Navier-Stokes equations and get some new conclusions. For one form of dynamics system with damping we prove the existence of solution, and get the existence of the attractors. Moreover, we discuss with limit-behavior the deformations of the Navier-Stokes equation.
\end{abstract}

Keywords: Incompressible Navier-Stokes Equation; Solution; Maximal Attractor; Limit-Behavior

\section{Introduction}

Concerned with the perturbed Navier-Stokes equations:

$$
\left\{\begin{array}{l}
u_{t}+(u \cdot \nabla) u-\sigma \Delta u+\nabla p=f+\alpha u, t>0, x \in \Omega, \\
\nabla u=0, \text { in } \Omega
\end{array}\right.
$$

where $\Omega \subset R^{2}$ is a smooth bounded domain with boundary $\Gamma \cdot u=\left(u_{1}, u_{2}\right)$, and $p, u$ is the velocity vector, $p(x, t)$ is the pressure at $x$ at time $t$, and $\sigma$ is the kinematic viscosity, and $\mathrm{f}$ represents volume forces that are applied to the fluid, and $0 \leq \alpha \leq \frac{\sigma}{4} \lambda_{1}$, where $\lambda_{1}$ is the first eigenvalue of $A$ (see Remark 4). The Equation (1.1) is Navier-Stokes equations, as $\alpha=0$, which show the existence of absorbing sets and the existence of a maximal attractor, the universal attractor, attractor in unbounded domain (see [1-5]). ACTA Mathematical Application Sinica. In [6], where they some interesting results, as $\beta=0$. In [7,8] Babin, Vishik and Abergel consider maximal attractors of semigroups corresponding to evolution differential equations, existence and finite dimensionality of global attractor for evolution equations on unbounded domains. In $[9,10]$ A. Pazy consider Semigroups of linear operator and application to partial differential equation.

\footnotetext{
*The research is supported by the Science Foundation of Baoshan University (No.13BY033).

"Corresponding author.
}

We need the following preliminaries:

Equations (1.1) are supplemented with a boundary condition. Two cases will be considered: The nonslip boundary condition. The boundary $\Gamma$ is solid and at rest; thus

$$
u=0 \in \Gamma^{1}
$$

The space-periodic case. Here $\Omega=\left(0, L_{1}\right) \times\left(0, L_{2}\right)$ and

$u, p$ the frist derivatives of $u$ are $\Omega$-periodic ${ }^{\text {Remark } 2}$.

Remark 1. If $\Gamma$ is solid but not rest, then the nonslip boundary condition is $u=\phi$ on $\Gamma$ where $\phi=\phi(x, t)$ is the give velocity of $\Gamma$.

Remark 2. That is $u$ and $p$ take the same values at corresponding points of $\Gamma$.

Furthermore, we assume in this case that the average flow vanishes

$$
\int_{\Omega} u \mathrm{~d} x=0 .
$$

When an initial-value problem is considered we supplement these equations with

$$
u(x, 0)=u_{0}(x), x \in \Omega .
$$

For the mathematical setting of this problem we consider a Hilbert space $H$ (see [8]) which is a close subspace of $L^{2}(\Omega)^{n} \quad(n=2$ here).

In the nonslip case, 


$$
H=\left\{u \in L^{2}(\Omega)^{n}, \operatorname{div} u=0, u \cdot v=0 \text { on } \Gamma\right\} .
$$

and in the periodic case

$$
H=\left\{u \in L^{2}(\Omega)^{n}, u_{i \mid \Gamma_{i}}=-u_{i \mid \Gamma_{i \pm n}}, i=1, \cdots, n\right\}^{\text {Remark } 3} .
$$

We refer the reader to $\mathrm{R}$. Temam [2] for more details on these spaces and, in particular, a trace theorem showing that the trace of $u \cdot v$ on $\Gamma$ exists and belong to $H^{-1 / 2}(\Gamma)$ when $u \in L^{2}(\Omega)^{n}$ and $\operatorname{div} u \in L^{2}(\Gamma)$. The space $H$ is endowed with the scalar product and the norm of $L^{2}(\Omega)^{n}$ denoted by $(\cdot, \cdot)$ and $\|\cdot\|$.

Remark 3. $\Gamma_{i}$ and $\Gamma_{i+n}$ are the faces $x_{i}=0$ and $x_{i}=L_{i}$ of $\Gamma$. The condition $u_{i \mid \Gamma_{i}}=-u_{i \mid \Gamma_{i+n}}$ expresses the periodicity of $u \cdot v ; L^{2}(\Omega)^{n}$ is the space of $u \in L^{2}(\Omega)^{n}$ satisfying (1.4).

Another useful space is $V$ a closed subspace of $H^{1}(\Omega)^{n}$

$$
V=\left\{u \in H^{1}(\Omega)^{n}, \operatorname{div} u=0\right\},
$$

in the nonslip case and , in the space-periodic case,

$$
V=\left\{u \in H_{\text {per }}^{\cdot 1}(\Omega)^{n}, \operatorname{div} u=0\right\},
$$

where $H_{p e r}$ is define in [1]. In both case, $v$ is endowed with the scalar product

$$
(u, v)=\sum_{i, j=1}^{n}\left(\frac{\partial u_{i}}{\partial x_{j}}, \frac{\partial v_{i}}{\partial x_{j}}\right)
$$

and the norm $\|u\|=(u, u)^{1 / 2}$.

We denoted by $A$ the linear unbounded operator in $H$ which is associated with $V, H$ and the scalar product $(u, v)=(A u, v), \forall u, v \in V$. The domain of $A$ in $H$ is denoted by $D(A)$; $A$ is self-adjoint positive operator in $H$. Also $A$ is an isomorphism from $D(A)$ onto $H$. The space $D(A)$ can be fully characterized by using the regularity theory of linear elliptic systems (see $[1,3]$ ).

$$
D(A)=H^{2}(\Omega)^{n} \cap V
$$

and $D(A)=H_{\text {per }}^{2}(\Omega)^{n} \cap V$ in the nonslip and periodic cases; furthermore, $|A u|$ is on $D(A)$ a norm equivalent to that induced by $H^{2}(\Gamma)^{n}$. Let $V^{\prime}$ be the dual of $V$; then $H$ can be identified to a subspace of $V^{\prime}$ and we have

$$
D(A) \subset V \subset H \subset V^{\prime},
$$

where the inclusions are continuous and each space is dense in the following one.

Remark 4. In the space-periodic case we have $A u=$ $-\Delta u, \forall u \in D(A)$, while in the nonslip case we have $A u=-P \Delta u, \forall u \in D(A)$, where $P$ is the orthogonal projector in $L^{2}(\Omega)^{n}$ on the space $H$. We can also say that $A u=f, u \in D(A), f \in H \quad$ is equivalent to saying that there exists $p \in H^{1}(\Omega)$ such that

$$
\begin{cases}-\Delta u+\operatorname{grad} p= & \text { in } \Omega, \\ \operatorname{div} u=0 & \text { in } \Omega, \\ u=0 & \text { in } \partial \Omega .\end{cases}
$$

The operator $A^{-1}$ is continuous from $H$ into $D(A)$ and since the embedding of $H^{1}(\Omega)$ in $L^{2}(\Omega)$ is compact, the embedding of $V$ in $H$ is compact. Thus $A^{-1}$ is a self-adjoint continuous compact operator in $H$, and by the classical spectral theorems there exists a sequence

$$
\lambda_{j}, 0<\lambda_{1} \leq \lambda_{2}, \cdots, \lambda_{j} \rightarrow \infty,
$$

and a family of elements $w_{j}$ of $D(A)$ which is orthonormal in $H$, and such that

$$
A w_{j}=\lambda_{j} w_{j}, \forall j \text {. }
$$

We need the following main Result:

Lemma 1.1. (see [4]) (Uniform Gronwall Lemma) Let $g, h, y$ be three positive locally integrable function on $\left[t_{0},+\infty\right]$ such that $y^{\prime}$ is locally integrable on $\left[t_{0},+\infty\right]$, and satisfy

$$
\begin{aligned}
& \frac{\mathrm{d} y}{\mathrm{~d} x} \leq g y+h t \text { for } t \geq t_{0} \\
& \int_{t}^{t+r}(g(s) \mathrm{d} s) \leq a_{1}, \int_{t}^{t+r}(h(s) \mathrm{d} s) \leq a_{2}, \int_{t}^{t+r}(y(s) \mathrm{d} s) \leq a_{3}
\end{aligned}
$$

for $t \geq t_{0}$ where $r, a_{1}, a_{2}, a_{3}$ are positive constant. Then

$$
y(t+r) \leq\left(\frac{a_{3}}{r}+a_{2}\right) \exp \left(a_{1}\right), \forall t \geq t_{0} .
$$

The evolution of the dynamical system is described by a family of operators $S(t), t \geq 0$, that map $H$ into itself and enjoy the usual semigroup properties (see [8]):

$$
\left\{\begin{array}{l}
S(t+s)=S(t) S(s), \forall s, t \geq 0 \\
S(0)=I,(\text { Indentify in } H) .
\end{array}\right.
$$

$S(T)$ is a continuous (nonlinear) operator from $H$ into itself $\forall t \geq 0$.

The operator $S(t)$ are uniformly compact for $t$ large. By this we mean that for every bounded set $X$ there exists $t_{0}$ which may depend on $X$ such that

$$
\bigcup_{t \geq t_{0}} S(t) X \text { is relatively compact in } H \text { (see [1]), }
$$

for every bounded set $C \subset H$,

$$
r_{c}(t)=\sup _{\phi \in C}\left|S_{2}(t) \phi\right| \rightarrow 0 \text { as } t \rightarrow \infty \text {. }
$$

Of course, if $H$ is Banach space, any family of operators satisfying (1.14) also satisfies (1.15) with $S_{2}=0$.

Theorem 1.2. (see [4]) We assume that $\mathrm{H}$ is a metric 
space and that the operators $S(t)$ are given and satisfy (1.12), (1.13) and either (1.14) or (1.15). We also assume that there exists an open set $\aleph$ and abounded set $X$ of $\aleph$ such that $X$ is absorbing in $\aleph$. Then $w$-limt set of $X, \Upsilon=w(X)$, is a compact attractor which attracts the bounded set of $\aleph$. It is the maximal bounded attractor in $\aleph$ (for the inclusion relation). Furthermore, if $H$ is a Banach space, if $U$ is convex ${ }^{2}$, and the mapping $t \rightarrow S(t) u_{0}$ is continuous from $R_{+}$into $H$, for every $u_{0}$ in $H$; then $\Upsilon$ is connected too.

The rest of this paper is organized such that Section 2 contains a sketch of existence and uniqueness of solution of the equations; in Section 3 we show the existence of absorbing set and the existence of a maximal attractor; in Section 4 contain the proof of existence and uniqueness of solution of the equations, in Section 5 discussed the perturbation coefficients $\alpha$.

\section{Existence and Uniqueness of Solution of the Equations}

The weak from of the Navier-Stokes equations due to J. Leray [1-3] involves only $u$, as $\alpha=0$. It is obtained by multiply (1.1) by a test function $v$ in $V$ and integrating over $\Omega$. Using the Green formula (1.1) and the boundary condition, we find that the term involving $p$ disappears and there remains

$$
\frac{\mathrm{d}}{\mathrm{d} t}(u, v)+\sigma(u, v)+b(u, u, v)=(f, v)+\alpha(u, v),
$$

where

$$
b(u, v, w)=\sum_{i, j=1}^{n} \int_{\Omega}\left(u_{i} \frac{\partial v_{j}}{\partial x_{i}} w_{j} \mathrm{~d} x\right),
$$

whenever the integrals make sense. Actually, the from $b$ is trilinear continuous on $H^{1}(\Omega)^{n}(n=2)$ and in particular on $V$. We have the following inequalities giving various continuity properties of $b$ :

$$
|b(u, v, w)| \leq c_{1} \times\left\{\begin{array}{c}
\|u\|_{H}^{\frac{1}{2}}\|A u\|_{H}^{\frac{1}{2}}\|v\|_{V}\|w\|_{H}, \\
\forall u \in D(A), v \in V, w \in H, \\
\|u\|_{H}^{\frac{1}{2}}\|u\|_{V}^{\frac{1}{2}}\|v\|_{V}\|w\|_{H}^{\frac{1}{2}}\|w\|_{V}^{\frac{1}{2}}, \\
\forall u, v, w \in V .
\end{array}\right.
$$

where $c_{1}>0$ is an appropriate constant[1].

An alternative from of (2.1) can be given using the operator $A$ and the bilinear operator $B$ from $V \times V$ into $V^{\prime}$ defined by

$$
(B(u, v), w)=b(u, v, w), \forall u, v, w \in V .
$$

we also set

$$
B(u)=B(u, u), \forall u \in V^{\prime},
$$

and we easily see that (2.1) is equivalent to the equation

$$
\frac{\mathrm{d} u}{\mathrm{~d} t}+\sigma A u+B(u)=f+\alpha u .
$$

while (1.5) can be rewritten

$$
u(0)=u_{0},
$$

We assume that $f$ is in dependent of $t$ so that the dynamical system associated with (2.5) is autonomous

$$
f(t)=f \in H, \forall t .
$$

Existence and uniqueness results for (2.5) (2.6) are well know as $\alpha=0$ (see [2,3]). The following theorem collects several classical results.

Theorem 1.3. Under the above assumption, for $f$ and $u_{0}$ given in $H, t$ here exists a unique solution $u$ of (2.4) (2.5) satisfying $u \in L^{\infty}([0, T] ; H) \cap L^{2}(0, t ; V)$; Furthermore, $u$ is analytic in $t$ with values in $D(A)$ for $t>0$, and the mapping $u_{0} \mapsto u(t)$ is continuous from $H$ into $D(A), \forall t>0$; Finally, if $u_{0} \in V$, then $u \in L^{\infty}([0, T] ; V) \cap L^{2}(0, T ; D(A)), \forall T>0$. Some indications for the proof of Theorem 1.3 will be given in Section 4. This theorem allows us to define the operators

$$
S(t): u_{0} \mapsto u(t)
$$

These operator enjoy the semigroup properties (1.12) and the are continuous from $H$ into itself and even from $H$ into $D(A)$.

\section{Absorbing Sets and Attractor}

The part proof about global attractor is similar to the Temam's book, but the exists of perturbation term is different from the Temam's book, so we reprove it for integrality.

Theorem 1.4. The dynamical system associated with the tow-dimensional modified Navier-Stokes equations, supplemented by boundary (1.2) or (1.3), (1.4) possesses an attractor $\chi$ that is compact, connected,and maximal in $H . \quad \chi \quad$ attracts the bounded sets of $H$ and $\chi$ is also maximal among the functional invariant set bounded in $H$.

Proof. We first prove the existence of an absorbing set in $H$. A first energy-type equality is obtained by taking the scalar product of (2.5) with $u$. Hence

$$
b(u, v, v)=0, \forall u \in V, \forall v \in H^{1}(\Omega)^{n},
$$

We see that $(B(u), u)=0$ and there remains

$$
\frac{1}{2} \frac{\mathrm{d}}{\mathrm{d} t}\|u\|_{H}^{2}+\sigma\|u\|_{V}^{2}=(f, u)+\alpha(u, u),
$$

We know that $\|u\|_{H} \leq \lambda_{1}^{-\frac{1}{2}}\|u\|_{V}$ where $\lambda_{1}$ is the first eigenvalue of $A$. Hence, we can majorize the right-hand 
side of (3.1) by

$$
(f, u) \leq\|f\|_{H}\|u\|_{H} \leq \frac{\sigma}{4}\|u\|_{V}^{2}+\frac{1}{\sigma \lambda_{1}}\|f\|_{H}^{2}
$$

the estimates $|\alpha(u, u)|$ :

$$
|\alpha(u, u)| \leq \alpha\|u\|_{H}^{2} \leq \frac{\alpha}{\lambda_{1}}\|u\|_{V}^{2} \leq \frac{\sigma}{4}\|u\|_{V}^{2}
$$

Hence we obtain

$$
\begin{aligned}
& \frac{\mathrm{d}}{\mathrm{d} t}\|u\|_{H}^{2}+\sigma\|u\|_{V}^{2} \leq \frac{1}{\sigma \lambda_{1}}\|f\|_{H}^{2}, \\
& \frac{\mathrm{d}}{\mathrm{d} t}\|u\|_{H}^{2}+\sigma \lambda_{1}\|u\|_{V}^{2} \leq \frac{1}{\sigma \lambda_{1}}\|f\|_{H}^{2},
\end{aligned}
$$

Using the classical Gronwall Lemma, we obtain

$$
\begin{aligned}
\|u(t)\|_{H}^{2} \leq & \left\|u_{0}\right\|_{H}^{2} \exp \left(-\lambda_{1} t\right) \\
& +\left(\frac{1}{\sigma^{2} \lambda_{1}^{2}}\|f\|_{H}^{2}\left(1-\exp \left(-\lambda_{1} t\right)\right)\right),
\end{aligned}
$$

Thus

$$
\limsup _{t \rightarrow \infty}\|u(t)\|_{H} \leq \rho_{0}, \rho_{0}=\frac{1}{\sigma \lambda_{1}}\|f\|_{H},
$$

We infer (3.5) that the ball $B_{H}(0, \rho)$ of $H$ with $\rho \geq \rho_{0}$ are positively invariants for the semigroup $S(t)$, and these balls are absorbing for any $\rho \geq \rho_{0}$. We choose $\left(\rho_{0}^{\prime}\right)>\rho_{0}$ and included a ball $B(0, R)$ of $H$, It is easy to deduce from (3.5) that $S(t) X \subset X B_{0}$ for $t\left(X, \rho_{0}^{\prime}\right)$, where

$$
t_{0}=\frac{1}{\sigma \lambda_{1}} \log \frac{R^{2}}{\left(\rho_{0}^{\prime}\right)^{2}-\left(\rho_{0}\right)^{2}} .
$$

We the infer from (3.3), after integration in $t$, that

$$
\sigma \int_{t}^{t+r}\|u\|_{V}^{2} \mathrm{~d} s \leq \frac{r}{\sigma \lambda_{1}}\|f\|_{H}^{2}+\|u(t)\|_{H}^{2}, \forall r>0,
$$

With the use of (3.6) we conclude that

$$
\limsup _{t \rightarrow \infty} \int_{t}^{t+r}\|u\|_{V}^{2} \mathrm{~d} s \leq \frac{r}{\sigma^{2} \lambda_{1}}\|f\|_{H}^{2}+\frac{1}{\sigma^{3} \lambda_{1}^{2}}\|u\|_{H}^{2},
$$

and if $u_{0} \in X \subset B_{H}(0, R)$ and $\left.t \geq t_{0} X, \rho_{0}^{\prime}\right)^{\prime}$, then

$$
\int_{t}^{t+r}\|u\|_{V}^{2} \mathrm{~d} s \leq \frac{r}{\sigma^{2} \lambda_{1}}\|f\|_{H}^{2}+\frac{1}{\sigma}\left(\rho_{0}^{\prime}\right)^{2},
$$

1) Absorbing set in $V$

An continue and show the existence of an absorbing set in $V$. For that purpose we obtain another energy-type equation by taking the scalar product of (2.5) with $A u$. Since

$$
\left(A u, u^{\prime}\right)=\left(u, u^{\prime}\right)=\frac{1}{2} \frac{\mathrm{d}}{\mathrm{d} t}\|u\|_{V}^{2},
$$

we find

$$
\frac{1}{2} \frac{\mathrm{d}}{\mathrm{d} t}\|u\|_{V}^{2}+\sigma\|A u\|_{H}^{2}+(B(u), A u)=(f, A u)+\alpha(u, A u),
$$

we writer

$$
\begin{aligned}
& \alpha(u, A u) \leq \alpha\|u\|_{H}\|A u\|_{H} \leq \frac{\sigma}{4}\|A u\|_{H}^{2}+\frac{\alpha^{2}}{\sigma}\|u\|_{H}^{2} \\
& (f, A u) \leq\|f\|_{H}\|A u\|_{H} \leq \frac{\sigma}{8}\|A u\|_{H}^{2}+\frac{2}{\sigma}\|f\|_{H}^{2},
\end{aligned}
$$

and using the second inequality (2.3)

$$
\begin{aligned}
|(B(u), A u)| & \leq c_{1}\|u\|_{H}^{\frac{1}{2}}\|u\|_{V}\|A u\|_{H}^{\frac{3}{2}} \\
& \leq \frac{\sigma}{4}\|A u\|_{H}^{2}+\frac{c_{1}^{\prime}}{\sigma^{3}}\|u\|_{H}^{2}\|u\|_{V}^{4} .
\end{aligned}
$$

Hence

$$
\begin{aligned}
& \frac{\mathrm{d}}{\mathrm{d} t}\|u\|_{V}^{2}+\sigma\|A u\|_{H}^{2} \\
& \leq\left[\frac{4}{\sigma}\|f\|_{H}^{2}+\frac{\alpha^{2}}{\sigma}\|u\|_{H}^{2}\right]+\frac{2 c_{1}^{\prime}}{\sigma^{3}}\|u\|_{H}^{2}\|u\|_{V}^{4},
\end{aligned}
$$

and since

$$
\|\phi\|_{L^{2}} \leq \lambda_{1}^{-\frac{1}{2}}\|A \phi\|_{L^{2}}, \forall \phi \in D(A)
$$

We also have

$$
\begin{aligned}
& \frac{\mathrm{d}}{\mathrm{d} t}\|u\|_{V}^{2}+\sigma \lambda_{1}\|A u\|_{H}^{2} \\
& \leq\left[\frac{4}{\sigma}\|f\|_{H}^{2}+\frac{\alpha^{2}}{\sigma}\|u\|_{H}^{2}\right]+\frac{2 c_{1}^{\prime}}{\sigma^{3}}\|u\|_{H}^{2}\|u\|_{V}^{4},
\end{aligned}
$$

We a priori estimate of $u L^{\infty}(0, T ; V), \forall T>0$, follows easily from (3.14) by the classical Gronwall lemma, using the previous estimates on $u$. We are more interested in an estimate valid for large $t$. Assuming that $u_{0}$ belong to a bounded set $X$ of $H$ and that $t \geq t_{0}\left(X, \rho_{0}^{\prime}\right), t_{0}$ as in (3.7), we apply the uniform Gronwall lemma to (3.14) with $g, h, y$ replaced by

$$
\frac{2 c_{1}^{\prime}}{\sigma^{3}}\|u\|_{H}^{2}\|u\|_{V}^{2}, \frac{4}{\sigma}\|f\|_{H}^{2}+\frac{\alpha^{2}}{\sigma}\|u\|_{H}^{2},\|u\|_{V}^{2},
$$

Thanks to (2.14), (2.18) we estimate the quantities $a_{1}, a_{2}, a_{3}$ in Lemma 1.1 by

$$
\left\{\begin{array}{l}
a_{1}=\frac{2 c_{1}^{\prime}}{\sigma^{3}}\left(\rho_{0}^{\prime}\right)^{2} a_{3}, \\
a_{2}=\frac{4 r}{\sigma}\|f\|_{H}^{2}+\frac{\alpha^{2}}{\sigma} \rho_{0}^{2}, \\
a_{3}=\frac{r}{\sigma^{2} \lambda_{1}}\|f\|_{H}^{2}+\frac{1}{\sigma}\left(\rho_{0}^{\prime}\right)^{2} .
\end{array}\right.
$$

and we obtain 


$$
\|u(t)\|_{V}^{2} \leq\left(\frac{a_{3}}{r}+a_{2}\right) \exp \left(a_{1}\right), \text { for } t \geq t_{0}+r,
$$

$t_{0}$ as in (3.7). Let us fix $r>0$ and denote by $\rho_{1}^{2}$ the right-hand side of (2.24). We the conclude that the ball $B\left(0, \rho_{1}\right)$ of $V$, denoted by $X_{1}$, is an absorbing set in $V$ for the semigroup $S(t)$. Furthermore, if $X$ is any bounded set of $H$, then $S(t) X \subset X_{1}$ for $t \geq t_{0}\left(X, \rho_{0}^{\prime}\right)+r$. This shows the existence of an absorbing set in $V$, namely $X$, and also that the operators $S(t)$ are uniformly compact, i.e., Theorem 1.1 is satisfied.

2) Maximal attractor

All the assumption of Theorem 1.1 are satisfied and we deduce from this theorem the existence of a maximal attractor for modified Navier-Stokes equations.

\section{Proof of Theorem 1.3}

The existence of a solution of (2.4) (2.5) that belong to $L^{\infty}(0, T ; H) \bigcap L^{2}(0, T ; V), \forall T>0$, is first obtain by the Faedo-Gakerkin (see [3]) method. We implement this approximation procedure with the function $w_{j}$ representing the eigenvalues of $A$ (see Remark 4). For each $m$ we look for an approximate solution $u_{m}$ of the form

$$
u_{m}(t)=\sum_{i=1}^{m} g_{i m}(t) w_{i}
$$

satisfying

$$
\begin{gathered}
\left(\frac{\mathrm{d} u_{m}}{\mathrm{~d} t}, w_{j}\right)+\sigma a\left(u_{m}, w_{j}\right)+b\left(u_{m}, u_{m}, w_{j}\right) \\
=\left(f, w_{j}\right)+\alpha\left(u_{m}, w_{j}\right), j=1, \cdots, m, \\
u_{m}=P_{m} u_{0},
\end{gathered}
$$

where $P_{m}$ is projector in $H$ (or $V$ ) on the space spanned by $w_{1}, \cdots, w_{m}$. Since $A$ and $P_{m}$ commute, the relation (3.1) is also equivalent to

$$
\frac{\mathrm{d} u^{m}}{\mathrm{~d} t}+\sigma A u_{m}+P_{m} B\left(u_{m}\right)=P_{m} f+\alpha P_{m} u_{m} .
$$

We prove $\left(u_{m}, w_{j}\right)$ on $g_{i m}$ is Lip continuous,

$$
h\left(u_{m}\right)=\sum_{i=1}^{m} g_{i m}(t) w_{i} w_{j} \text {. }
$$

hence

$$
\left|h\left(g_{i m}\right)-h\left(\bar{g}_{i m}\right)\right|=\left|\sum_{i=1}^{m} g_{i m}(t) w_{i} w_{j}-\sum_{i=1}^{m} \bar{g}_{i m}(t) w_{i} w_{j}\right|
$$

there is $m, M$ such that $m \leq\left|g_{i m}(t)\right| \leq M,\left|w_{i}\right|=1$. When $i=j, w_{i} \cdot w_{j}=\left|w_{i}\right|^{2}=1$ is established, and when $i \neq j, w_{i} \cdot w_{j}=0$ is established, then

$$
\left|h\left(g_{i m}\right)-h\left(\bar{g}_{i m}\right)\right| \leq C\left|g_{i m}(t)-\bar{g}_{i m}(t)\right| \text {. }
$$

On both sides in the integral $\Omega$, then

$$
\begin{aligned}
\int_{\Omega}\left|h\left(g_{i m}\right)-h\left(\bar{g}_{i m}\right)\right| \mathrm{d} x & \leq \int_{\Omega} C\left|g_{i m}(t)-\bar{g}_{i m}(t)\right| \mathrm{d} x \\
& \leq C\left|g_{i m}(t)-\bar{g}_{i m}(t)\right| .
\end{aligned}
$$

we writer

$$
\left|\left(u_{m}, w_{j}\right)-\left(\bar{u}_{m}, w_{j}\right)\right| \leq C\left|g_{i m}(t)-\bar{g}_{i m}(t)\right| .
$$

Hence $\left(u_{m}, w_{j}\right)$ on $g_{i m}$ is Lip continuous. The existence and uniqueness of $u_{m}$ on some interval $\left[0, T_{m}\right]$ is elementary and then $T_{m}=+\infty$, because of the a priori estimates that we obtain for $u_{m}$. An energy equality is obtained by multiplying (4.1) by $g_{j m}$ and summing these relations for $j=1, \cdots, m$. We obtain (3.2) exactly with $u$ replaced by $u_{m}$ and we deduce from this relation that

$u_{m}$ remains bounded in $L^{\infty}(0, T ; H) \cap L^{2}(0, T ; V)$, $\forall T>0$.

Due to (3.1) and the last inequality (2.3)

$$
\|B(\varphi)\|_{V} \leq\|\varphi\|_{H}\|\varphi\|_{V}, \forall \varphi \in V \text {. }
$$

Therefor $B\left(u_{m}\right)$ and $P_{m} u_{m}$ remain bounded in $L^{2}\left(0, T ; V^{\prime}\right)$ and by (4.3)

$$
\frac{\mathrm{d} u_{m}}{\mathrm{~d} t} \text { remains bounded in } L^{2}\left(0, T ; V^{\prime}\right) \text {. }
$$

By weak compactness it follows from (4.3) that there exists $u \in L^{\infty}(0 ; T ; H) \cap L^{2}(0, T ; V), \forall t>0$, and a subsequence still denoted $m$, such that

$$
\begin{aligned}
& u_{m} \rightarrow u \in L^{2}(0, T ; V) \text { weakly and in } L^{\infty}(0, T ; H) \\
& \text { weak-star } \frac{\mathrm{d} u_{m}}{\mathrm{~d} t} \rightarrow \frac{\mathrm{d} u}{\mathrm{~d} t} \text { in } L^{2}\left(0, T ; V^{\prime}\right) \text { weakly. }
\end{aligned}
$$

Due to (4.6) and a classical compactness theorem (see [2]), we also have

$$
u_{m} \rightarrow u \text {,in } L^{2}(0, T ; H) \text { strongly. }
$$

This is sufficient to pass to the limit in (4.1)-(4.3) and we find (2.4), (2.5) at the limit. For (2.5) we simply observe that (4.7) implies that $u_{m}(t) \rightarrow u(t)$ weakly in $V^{\prime}$ or even in $H, \forall t \in[0, T]$.

By (2.4), $u^{\prime}=\frac{\mathrm{d} u}{\mathrm{~d} t}$ belong to $L^{2}\left[(0, T) ; V^{\prime}\right]$ and, $u$ is in $L^{\infty}([0, T] ; H)$. The uniqueness and continuous dependent of $u(t)$ on $u_{0}$ (in $H$ ) follow by standard using [2].

The fact that $u \in L^{\infty}(0, T ; V) \cap L^{2}(0, T ; D(A)), \forall T>0$, is proved by deriving further a priori estimates on $u_{m}$. They are obtained by multiplying (4.1) by $\lambda_{j} g_{j m}$ and summing these relations for $j=1, \cdots, m$. Using (1.11) we find a relation that is exactly (3.11) with $u$ replaced by $u_{m}$. we deduce form this relation that 
$u_{m}$ remains bounded $\in L^{\infty}(0, T ; V) \cap L^{2}(0, T ; D(A))$,

$\forall T>0$.

At the limit we then find that $u$ is in $L^{2}(0, T: D(V))$. The fact that $u$ in $L^{\infty}([0, T], V)$ then follows from an appropriate application of Lemma 3.2 [2].

Finally, the fact that $u$ is analytic in $t$ with values in $D(A)$ results from totally different methods, for which the reader is referred to C. Foias and R. Temam [1] or R. Temam [3]. However, this property was given for the sake of completness and is never used here in an essential manner.

\section{The Limit-Behavior of Navier-Stokes Equation with Nonlinear Perturbation}

We consider the limit-behavior of Navier-Stokes equation with nonlinear perturbation on the two dimensional space. we use the space which is given (1.6), (1.8). The main advantage we see is that applying the Gronwall lemma to the solution of problem (1.1) approaches a solution of Navier-Stokes equation on $L^{2}$ and $H^{2}$, as $\alpha \rightarrow 0$.

Theorem 1.6. Under assumption (1.6), then the solution of $u(x, t, \alpha)$ of (1.1) is approximate solution of Navier-Stokes equations and this solution is stable, as $\alpha \rightarrow 0$.

Proof. Let $u=\left(x, t, \alpha_{1}\right)=u\left(\alpha_{1}\right)$ is a solution of (1.1), as $\alpha=\alpha_{1}$ :

$$
\left\{\begin{array}{l}
u_{t}+(u \cdot \nabla) u-\sigma \Delta u+\nabla p=f+\alpha_{1} u, t>0, x \in \Omega, \\
\nabla u=0, \text { in } \Omega, \\
u(0)=u_{0}
\end{array}\right.
$$

Let $u=\left(x, t, \alpha_{2}\right)=u\left(\alpha_{2}\right)$ is a solution of (1.1), as $\alpha=\alpha_{2}$ :

$$
\left\{\begin{array}{l}
u_{t}+(u \cdot \nabla) u-\sigma \Delta u+\nabla p=f+\alpha_{2} u, t>0, x \in \Omega, \\
\nabla u=0, \text { in } \Omega, \\
u(0)=u_{0}
\end{array}\right.
$$

Utilizing (5.1)-(5.2) and let $v=u\left(\alpha_{1}\right)-u\left(\alpha_{2}\right)$, Hence

$$
\begin{aligned}
& v_{t}+\left[\left(u\left(\alpha_{1}\right) \cdot \nabla\right) u\left(\alpha_{1}\right)-\left(u\left(\alpha_{2}\right) \cdot \nabla\right) u \alpha_{2}\right]-\sigma \Delta v \\
& =\alpha_{1} u\left(\alpha_{1}\right)-\alpha_{2} u\left(\alpha_{2}\right) .
\end{aligned}
$$

It is obtained by multiply (5.4) by a function $v$ in $V$ and integrating over $\Omega$.

$$
\begin{aligned}
& \frac{1}{2} \frac{\mathrm{d}}{\mathrm{d} t}\|v\|_{H}^{2}+\sigma\|v\|_{V}^{2} \\
& +\int_{\Omega}\left[\left(u\left(\alpha_{1}\right) \cdot \nabla\right) u\left(\alpha_{1}\right)-\left(u\left(\alpha_{2}\right) \cdot \nabla\right) u\left(\alpha_{2}\right)\right] v \mathrm{~d} x \\
& =\int_{\Omega}\left[\alpha_{1} u\left(\alpha_{1}\right)-\alpha_{2} u\left(\alpha_{2}\right)\right] v \mathrm{~d} x .
\end{aligned}
$$

Using the second inequality (2.3) and $b(u, v, w)$ is trilinear continuous:

$$
\begin{aligned}
& \int_{\Omega}\left[\left(u\left(\alpha_{1}\right) \cdot \nabla\right) u\left(\alpha_{1}\right)-\left(u\left(\alpha_{2}\right) \cdot \nabla\right) u\left(\alpha_{2}\right)\right] v \mathrm{~d} x \\
& =\left|b\left(u\left(\alpha_{2}\right), u\left(\alpha_{2}\right), v\right)-b\left(u\left(\alpha_{1}\right), u\left(\alpha_{1}\right), v\right)\right| \\
& \leq\left|b\left(u\left(\alpha_{2}\right), u\left(\alpha_{2}\right), v\right)-b\left(u\left(\alpha_{1}\right), u\left(\alpha_{2}\right), v\right)\right| \\
& \quad+\left|b\left(u\left(\alpha_{1}\right), u\left(\alpha_{1}\right), v\right)-b\left(u\left(\alpha_{1}\right), u\left(\alpha_{1}\right), v\right)\right| \\
& \leq\left|b\left(v, u\left(\alpha_{2}\right), v\right)-b\left(u\left(\alpha_{1}\right), v, v\right)\right| \\
& \leq c_{1}^{\prime}\|v\|_{H}^{\frac{1}{2}}\|v\|_{V}^{\frac{1}{2}}\left\|u\left(\alpha_{2}\right)\right\|_{V}\|v\|_{H}^{\frac{1}{2}}\|v\|_{V}^{\frac{1}{2}} \\
& \quad+c_{1}^{\prime \prime}\left\|u\left(\alpha_{2}\right)\right\|_{H}^{\frac{1}{2}}\left\|u\left(\alpha_{2}\right)\right\|_{V}^{\frac{1}{2}}\|v\|_{V}\|v\|_{V}^{\frac{1}{2}}\|v\|_{H}^{\frac{1}{2}} \\
& \leq c_{1}\|v\|_{V}^{2},
\end{aligned}
$$

and

$$
\begin{aligned}
& \int_{\Omega}\left[\alpha_{1} u\left(\alpha_{1}\right)-\alpha_{2} u\left(\alpha_{2}\right)\right] v \mathrm{~d} x \\
& =\left|\alpha_{1}\left(u\left(\alpha_{1}\right), v\right)-\alpha_{2}\left(u\left(\alpha_{2}\right), v\right)\right| \\
& \leq\left|\alpha_{1}\left(u\left(\alpha_{1}\right), v\right)-\alpha_{2}\left(u\left(\alpha_{1}\right), v\right)\right| \\
& \quad+\left|\alpha_{2}\left(u\left(\alpha_{1}\right), v\right)-\alpha_{2}\left(u\left(\alpha_{2}\right), v\right)\right| \\
& \leq\left|\alpha_{1}-\alpha_{2}\right|\left(u\left(\alpha_{1}\right), v\right)|+| \alpha_{2}||\left(u\left(\alpha_{1}\right)-u\left(\alpha_{2}\right), v\right) \mid,
\end{aligned}
$$

utilizing inequality (**), (3.6), (3.46) we estimate

$\left(\left|\operatorname{Du}\left(\alpha_{1}\right)\right|^{\beta} u\left(\alpha_{1}\right), v\right)$ :

$$
\begin{aligned}
\left|\left(u\left(\alpha_{1}\right), v\right)\right| & =\left|\int_{\Omega} u\left(\alpha_{1}\right) v \mathrm{~d} x\right| \leq \int_{\Omega} u\left(\alpha_{1}\right)\left|u\left(\alpha_{1}\right)-u\left(\alpha_{2}\right)\right| \mathrm{d} x \\
& \leq \int_{\Omega}\left|u\left(\alpha_{1}\right)\right|^{2} \mathrm{~d} x+\int_{\Omega}\left|u\left(\alpha_{1}\right)\right|\left|u\left(\alpha_{2}\right)\right| \mathrm{d} x \\
& \leq\left\|u\left(\alpha_{1}\right)\right\|_{V}^{2}+\left\|u\left(\alpha_{1}\right)\right\|_{H}\left\|u\left(\alpha_{2}\right)\right\|_{H}=c_{2},
\end{aligned}
$$

we write

$$
\begin{aligned}
& \left|\left(u\left(\alpha_{1}\right)-u\left(\alpha_{2}\right), v\right)\right| \\
& =|(v, v)|=\left|\int_{\Omega} v^{2} \mathrm{~d} x\right| \leq \int_{\Omega}|v|^{2} \mathrm{~d} x \leq c_{3} \|\left. v\right|_{V} ^{2},
\end{aligned}
$$

hence

$$
* * * \leq c_{2}\left|\alpha_{1}-\alpha_{2}\right|+c_{3}\|v\|_{V}^{2} .
$$

where $c_{1}>0, c_{2}>0, c_{3}>0$ is an appropriate constant. Hence

$$
\frac{1}{2} \frac{\mathrm{d}}{\mathrm{d} t}\|v\|_{H}^{2}+\sigma\|v\|_{V} \leq c_{2}\left|\alpha_{1}-\alpha_{2}\right|+\left(c_{1}+c_{3}\right)\|v\|_{V}^{2} .
$$

i.e.

$$
\frac{\mathrm{d}}{\mathrm{d} t}\|v\|_{H}^{2} \leq 2 c_{2}\left|\alpha_{1}-\alpha_{2}\right|+2\left(c_{1}+c_{3}\right)\|v\|_{V}^{2} .
$$

Using the classical Gronwall Lemma, we obtain 


$$
\|v\|_{H}^{2} \leq\left\|v_{0}\right\|_{V} \exp \left(-C_{2} t\right)+\frac{C_{1}}{C_{2}}\left(1-\exp \left(-C_{2} t\right)\right),
$$

where

$$
C_{1}=2 c_{2}\left|\alpha_{1}-\alpha_{2}\right|, C_{2}=2\left(c_{1}+c_{3}\right) .
$$

Notice that $v_{0}=0$. Thanks to

$$
\lim _{\alpha_{1} \rightarrow \alpha_{2}} C_{1}=0
$$

hence

$$
\lim _{\alpha_{1} \rightarrow \alpha_{2}} v=0
$$

Hence $C_{1}=0, C_{2}>0$ as $\alpha_{1}=\alpha_{2}=\alpha$, according to stable condition, thus this solution is stable.

\section{REFERENCES}

[1] C. Foias and R. Teman, "Attractor Representing Tulent Flows," Memoirs of Applied Mathematical Sciences, Vol. 53, No. 314, 1985.

[2] C. Foias and R. Teman, "On the Dimension of the Attractors in Two-Demensional Turbulence," Physica D, Vol. 30, No. 3, 1988, pp. 284-296. doi:10.1016/0167-2789(88)90022-X

[3] C. Foias and R. Teman, "On the Large-Time Galerkin Approximation of the Navier-Stokes Equations," SIAM
Journal on Numerical Analysis, Vol. 21, No. 4, 1984, pp. 615-634. doi:10.1137/0721043

[4] J. E. Marsden, L. Sirovich and F. John, "Infinite-Dimensional Dynamical Systems in Mechanics and Physics," Applied Mathematical Sciences, Vol. 68, 1997, SpringerVerlag, New York, pp. 15-25.

[5] F. Abergel, "Attractor for a Navier-Stokes Flow in Unbounded Domain," Mathematical Modelling and $\mathrm{Nu}$ merical Analysis, Vol. 23, No. 3, 1989, pp. 359-370.

[6] C. S. Zhao and K. T. Li, “The Global Attractor of N-S Equation with Linear Dampness on the Whole TwoDimensional Space and Estimates of Its Demensions," ACTA Mathematical Application Sinica, Vol. 23, No. 1, 2000, pp. 90-96.

[7] A. V. Babin and M. I. Vishik, "Maximal Attractors of Semigroups Corresponding to Evolution Differential Equations,” Mathematics of the USSR-Sbornik, Vol. 54, No. 2, 1986, pp. 387-408. doi:10.1070/SM1986v054n02ABEH002976

[8] F. Abergel, "Existence and Finite Dimensionality of Global Attractor for Evolution Equations on Unbounded Domains,” Journal of Differential Equations, Vol. 83, No. 1, 1990, pp. 85-108. doi:10.1016/0022-0396(90)90070-6

[9] R. S. Adms, “Sobolve Space,” Academic Press, New York, 1975.

[10] A. Pazy, "Semigroups of Linear Operator and Application to Partial Differential Equation," Applied Mathematical Sciences, Springer-Verlag, New York, 2006, pp. 1-38. 